\title{
Qualitative Behavior of Solutions to a Mathematical Model of Memes Transmission
}

\author{
Reem Al-Amoudi, Sarah Al-Sheikh, Salma Al-Tuwairqi * \\ Department of mathematics, King Abdulaziz university, Jeddah 21551, Saudi Arabia \\ *Corresponding author E-mail: saltuwairqi@kau.edu.sa
}

Copyright (c) 2014 Reem Al-Amoudi et. al. This is an open access article distributed under the Creative Commons Attribution License, which permits unrestricted use, distribution, and reproduction in any medium, provided the original work is properly cited.

\begin{abstract}
Researchers have applied epidemiological models to study the dynamics of social and behavioral processes, based on the fact that both biological diseases and social behavioral are a result from interactions between individuals. The main feature of the paper is to understand the dynamics of spreading a meme on a large scale in a short time through a chain of communications. In this paper we study a meme transmission model, which is an extension of the deterministic Daley-Kendall model and we analyze it by using stability theory of nonlinear differential equations. The model is based on dividing the population into three disjoint classes of individuals according to their reaction to the meme. We examine the existence of equilibria of the model and investigate their stability using linearization methods, Lyapunov method and Hopf bifurcation analysis. One of the significant results in this paper is finding conditions that will lead to persistent of memes. Also numerical simulations are used to support the results.
\end{abstract}

Keywords: Basic reproduction number, Global stability, Hopf bifurcation, Local stability, Lyapunov function, Memes transmission model.

\section{Introduction}

The dynamics of real life systems may be explained by a mathematical model. A model gives an inside picture of the different components of the system and the relation between them. Through analyzing the model one may give certain predictions of behavior of the system.

Extensive research has been going on for decades in modeling biological diseases and epidemics systems. Later on, Researchers have applied epidemiological models to study the dynamics of other social systems. In particular, the dynamics of rumors spread and transmission of ideas and thoughts. This was based on the fact that both biological diseases and social behavior are a result from interactions between individuals.

A rumor or an idea is like a virus, once it is transmitted to an individual, an outbreak of events happen in a short period of time. Spreading a rumor is like a contagious disease that does not need more than a few infected individuals to infect the entire population. If a rumor starts in a population, eventually everyone will know it.

Daley and kendall are among the earliest researchers to propose a rumor spread model that has some properties in common with epidemic model [1]. Also Cane [2] showed that deterministic forms of models for the spread of an epidemic and of a rumor are similar. Recently, Thompson et al. [3] explored the dynamics of rumor spreading in chat rooms. Bettencourt et al. [4] applied models similar to epidemiology to the spread of ideas. Kawachi [5] proposed deterministic models for rumor transmission with constant and variable rumor in an age-independent 
population. Kawachi et al. [6] explored the effects of various contact interactions in a rumor transmission model. Piqueira [7] examined an equilibrium study of a rumor spreading model according to propagation parameters and initial conditions. Huang [8] studied the rumor spreading process with denial and skepticism. Wang and Wood [9] adopted an epidemiological approach to model viral meme propagation. Meme, as defined in their paper, is any cultural entity that appears to exhibit self-replication. Huo et al. [10] analyzed the dynamics of a rumor transmission model with incubation. Zhao et al. [11] proposed a mathematical model for exploring the interplay of authorities, information, rumor dissemination and the evolution of emergency, aiming to give more insights on effective strategies for rumor management.

The significant of studying the dynamics of transmission of rumors and ideas lies in understanding a way to change perception, to persuade others, to generate predictions regarding product adoption, to predict what the public may find interesting in the future, and to influence decision making and public opinion.

There are different ways to approach a mathematical model. One of these is a qualitative approach, which involves the study of long term behavior of solutions, local and global stability of steady state (equilibrium) solutions, periodicity of solutions, and other behaviors.

In this paper, we study a qualitative behavior of a model of a constant rumor transmission in a population with constant immigration and emigration. This model was proposed by Kawachi [5]. He studied the limit system of the model that is, reducing the model to a system of two ordinary differential equations. Here, we investigate and give a comprehensive qualitative study for the full system of the model . The mathematical model and basic reproduction number is described in section 2. Equilibria of the model are identified in section 3. Section 4 analyzes the stability of equilibria both locally and globally using linearization methods and Lyapunov method. Further analysis using Hopf bifurcation is demonstrated in section 5. Numerical simulations are illustrated in section 6. Discussions and conclusions are given in Section 7 .

\section{Mathematical model and basic reproduction number}

Let $N(t)$ denote the total population. We divided the population into three disjoint classes of individuals:

$S(t)$ : the susceptible class, describing people who have not yet been exposed to a particular meme.

$I(t)$ : the spreader class, referring to people who take an active interest in the idea or concept that a meme represents, and therefore have a tendency to talk about the meme in social interactions.

$Z(t)$ : the stifler, meaning people who have experienced the meme, but have no interest in it or have lost interest at some point.

Note that $N(t)=S(t)+I(t)+Z(t)$. Taking the above considerations, the model dynamics is assumed by the following nonlinear system of ordinary differential equations:

$$
\begin{aligned}
\frac{d S}{d t} & =B-\alpha S I-\mu S \\
\frac{d I}{d t} & =\alpha \theta S I-\beta I^{2}-\gamma I Z-\mu I \\
\frac{d Z}{d t} & =\alpha(1-\theta) S I+\beta I^{2}+\gamma I Z-\mu Z
\end{aligned}
$$

where the positive parameters are defined as follows:

$B$ is the birth rate.

$\mu$ is the death rate.

$\alpha$ is the rate at which susceptibles change their meme class, where $\alpha=c q$ such that $c$ is the average number of contact per unit time and $q$ is the probability of transmitting the meme.

$\beta$ is the rate at which spreaders become stiflers by contacting with each other.

$\gamma$ is the rate at which spreaders become stiflers by contacting with stiflers.

$\theta$ is the fraction of susceptibles who become spreaders (at rate $\alpha$ ).

$1-\theta$ is the fraction of susceptibles who become stiflers (at rate $\alpha$ ), such that $\theta \in(0,1]$.

It follows from system (1) that $\frac{d N}{d t}+\mu N=B$, which can be solved easily to give 
$N(t)=N_{0} \exp (-\mu t)+\frac{B}{\mu}[1-\exp (-\mu t)]$, where $N_{0}=N(0)$, and therefore,

$\lim _{t \rightarrow \infty} N(t)=\frac{B}{\mu}$

Thus, the considered region for system (1) is

$\Gamma=\left\{(S, I, Z): S+I+Z \leq \frac{B}{\mu}, S>0, I \geq 0, Z \geq 0\right\}$.

$\Gamma$ is positively invariant since all solutions of system (1) in $\Gamma$ remain there for all $t>0$.

The basic reproduction number $R_{0}$ for system (1) is found using the method of next generation matrix [12]. But first we obtain the meme free equilibrium $E_{0}$. Consider the equations:

$$
\begin{aligned}
B-\alpha S I-\mu S & =0 \\
\alpha \theta S I-\beta I^{2}-\gamma I Z-\mu I & =0 \\
\alpha(1-\theta) S I+\beta I^{2}+\gamma I Z-\mu Z & =0 .
\end{aligned}
$$

Let $I=Z=0$ in $(2)$, then $E_{0}=\left(\frac{B}{\mu}, 0,0\right)$. Now let $X=(I, Z, S)$, then system $(1)$ may be written as: $X^{\prime}=\mathcal{F}(X)-\mathcal{V}(X)$,

where $\mathcal{F}(X)=\left[\begin{array}{c}\alpha \theta S I \\ \alpha(1-\theta) S I \\ 0\end{array}\right], \mathcal{V}(X)=\left[\begin{array}{c}\beta I^{2}+\gamma I Z+\mu I \\ -\beta I^{2}-\gamma I Z+\mu Z \\ -B+\alpha S I+\mu S\end{array}\right]$.

The Jacobian matrices of $\mathcal{F}(X)$ and $\mathcal{V}(X)$ at the meme free equilibrium point $E_{0}$, are $D \mathcal{F}\left(E_{0}\right)=\left[\begin{array}{ll}F & 0 \\ 0 & 0\end{array}\right]$ and $D \mathcal{V}\left(E_{0}\right)=\left[\begin{array}{cc}V & 0 \\ J_{1} & J_{2}\end{array}\right]$,

where $F=\left[\begin{array}{cc}\alpha \theta S_{0} & 0 \\ \alpha(1-\theta) S_{0} & 0\end{array}\right] V=\left[\begin{array}{cc}\mu & 0 \\ 0 & \mu\end{array}\right], J_{1}=\left[\begin{array}{ll}\alpha S_{0} & 0\end{array}\right], J_{2}=[\mu]$.

The next generation matrix is $F V^{-1}=\left[\begin{array}{cc}\frac{\alpha \theta B}{\mu^{2}} & 0 \\ \frac{\alpha(1-\theta) B}{\mu^{2}} & 0\end{array}\right]$. $\frac{\alpha \theta B}{\mu^{2}}$.

The basic reproduction number of system (1) is the spectral radius of matrix $F V^{-1}$, that is, $R_{0}=\rho\left(F V^{-1}\right)=$

\section{Equilibria of the model}

Besides the meme free equilibrium $E_{0}$ obtained in previous section, solutions of (2) give rise to two positive (meme existence) equilibriums: $E_{1}^{*}=\left(S^{*}, I_{1}^{*}, Z^{*}\right)$ and $E_{2}^{*}=\left(S^{*}, I_{2}^{*}, Z^{*}\right)$. Here

$$
\begin{aligned}
S^{*} & =\frac{B}{\alpha I^{*}+\mu}, \\
Z^{*} & =\frac{-\mu^{2}}{\gamma\left(\alpha I^{*}+\mu\right)}\left[\frac{\beta \alpha I^{*^{2}}}{\mu^{2}}+\frac{\beta I^{*}}{\mu}+\frac{\alpha I^{*}}{\mu}+1-R_{0}\right],
\end{aligned}
$$

and $I_{1}^{*}, I_{2}^{*}$ are the roots of the equation $a I^{*^{2}}+b I^{*}+c=0$, where

$$
\begin{aligned}
& a=\alpha \mu\left(\frac{\beta}{\gamma}-1\right) \\
& b=\alpha(1-\theta) B+\mu^{2}\left(R_{0}-1\right)+\frac{\mu^{2} \beta}{\gamma}+\frac{\mu^{2} \alpha}{\gamma}=\gamma \alpha B+\alpha \mu^{2}+\mu^{2}(\beta-\gamma), \\
& c=\frac{\mu^{3}}{\gamma}\left(1-R_{0}\right) .
\end{aligned}
$$

Note that: 
- $Z^{*}$ does not exist biologically when $R_{0} \leq 1$.

- $a$ is positive when $\beta>\gamma$, and negative when $\beta<\gamma$.

- $b$ is positive when either $R_{0}>1$ or $\beta>\gamma$.

- $c$ is positive when $R_{0}<1$, and negative when $R_{0}>1$.

Thus, when $R_{0}>1$ we have two cases for the roots $I_{1}^{*}, I_{2}^{*}$ :

case 1: for $\beta>\gamma$ one positive root exists $I_{1}^{*}=\frac{-b+\sqrt{b^{2}-4 a c}}{2 a}$.

case 2: for $\beta<\gamma$ two positive roots exists if $b^{2}-4 a c>0, I_{1}^{*}=\frac{-b+\sqrt{b^{2}-4 a c}}{2 a}$ and $I_{2}^{*}=\frac{-b-\sqrt{b^{2}-4 a c}}{2 a}$.

We will define $R_{1}=\frac{\gamma}{\beta}$, and state the following theorem for the existence of the equilibria .

Theorem 1 System (1) has the following equilibria:

- The meme free equilibrium $E_{0}=\left(\frac{B}{\mu}, 0,0\right)$ which exists always.

- If $R_{0}>1$ and $R_{1}<1$, we have a unique positive (meme existence) equilibrium

$E_{1}^{*}=\left(S^{*}, I_{1}^{*}, Z^{*}\right)$

- If $R_{0}>1$ and $R_{1}>1$, we have two positive (meme existence) equilibrium

$E_{1}^{*}=\left(S^{*}, I_{1}^{*}, Z^{*}\right), E_{2}^{*}=\left(S^{*}, I_{2}^{*}, Z^{*}\right)$ if $b^{2}-4 a c>0$.

We may think of $R_{0}$ and $R_{1}$ as bifurcation parameters since the number of equilibria changes as we cross the values $R_{0}=1$ and $R_{1}=1$.

\section{Stability of the equilibria}

\subsection{Local stability}

In this subsection we investigate the local stability of meme free equilibrium $E_{0}$ and positive (meme existence) equilibrium $E^{*}$. We state the following theorems:

Theorem 2 (local stability of $E_{0}$ ) If $R_{0}<1$, the meme free equilibrium $E_{0}$ of system (1) is locally asymptotically stable. If $R_{0}=1, E_{0}$ is locally stable. If $R_{0}>1, E_{0}$ is unstable.

Proof: By linearization method [13], the Jacobian matrix of system (1) at the meme free equilibrium $E_{0}$ is:

$$
J\left(E_{0}\right)=\left[\begin{array}{ccc}
-\mu & \frac{-\alpha B}{\mu} & 0 \\
0 & \frac{\alpha \theta B}{\mu}-\mu & 0 \\
0 & \frac{\alpha(1-\theta) B}{\mu} & -\mu
\end{array}\right] .
$$

Clearly the eigenvalues of $J\left(E_{0}\right)$ are: $\lambda_{1,2}=-\mu<0$, and $\lambda_{3}=\mu\left(R_{0}-1\right)<0$ if $R_{0}<1$. Hence, $E_{0}$ is locally asymptotically stable if $R_{0}<1$. If $R_{0}=1$ then the eigenvalues are $\lambda_{1,2}=-\mu<0$, and $\lambda_{3}=0$. So, $E_{0}$ is locally stable. If $R_{0}>1, E_{0}$ is unstable since $\lambda_{3}>0$.

Theorem 3 (local stability of $E^{*}$ ) The positive(meme existence)equilibrium $E^{*}$ of system (1) is locally asymptotically stable if $R_{1}<1$.

Proof: Linearization of system $(1)$ at $E^{*}=\left(S^{*}, I^{*}, Z^{*}\right)$ gives:

$$
J\left(E^{*}\right)=\left[\begin{array}{ccc}
\frac{-B}{S^{*}} & -\alpha S^{*} & 0 \\
\alpha \theta I^{*} & -\beta I^{*} & -\gamma I^{*} \\
\alpha(1-\theta) I^{*} & \beta I^{*}+\frac{\mu Z^{*}}{I^{*}} & \gamma I^{*}-\mu
\end{array}\right] .
$$


The eigenvalues of $J\left(E^{*}\right)$ are the roots of the equation $\lambda^{3}+a_{1} \lambda^{2}+a_{2} \lambda+a_{3}=0$, where

$a_{1}=\frac{B}{S^{*}}+(\beta-\gamma) I^{*}+\mu$.

$a_{2}=\alpha^{2} \theta S^{*} I^{*}+\frac{B}{S^{*}}(\beta-\gamma) I^{*}+\mu \frac{B}{S^{*}}+\beta \mu I^{*}+\gamma \mu Z^{*}$.

$a_{3}=\mu \frac{B}{S^{*}}\left(\beta I^{*}+\gamma Z^{*}\right)+\alpha^{2}\left(\mu \theta-\gamma I^{*}\right) S^{*} I^{*}$.

Clearly $a_{1}, a_{2}>0$ when $\beta>\gamma$. Moreover, since $S^{*}=\frac{\mu(\beta-\gamma) I^{*}+\mu^{2}}{\alpha \mu \theta-\gamma \alpha I^{*}}>0$ and $\beta>\gamma$, then $\mu \theta-\gamma I^{*}>0$, thus $a_{3}>0$.

$$
\begin{aligned}
& a_{1} a_{2}-a_{3}=\alpha^{2} \gamma S^{*} I^{*^{2}}+\frac{B}{S^{*}} \alpha^{2} \theta S^{*} I^{*}+\frac{B^{2}}{S^{* 2}}(\beta-\gamma) I^{*}+\mu \frac{B^{2}}{S^{2}}+\mu \frac{B}{S^{*}}(\beta-\gamma) I^{*}+\mu^{2} \frac{B}{S^{*}} \\
& +\beta \mu^{2} I^{*}+\gamma \mu^{2} Z^{*}+\left((\beta-\gamma) I^{*}\right)\left(\alpha^{2} \theta S^{*} I^{*}+\frac{B}{S^{*}}(\beta-\gamma) I^{*}+\mu \frac{B}{S^{*}}+\beta \mu I^{*}+\gamma \mu Z^{*}\right)>0 \text { if } \beta>\gamma \text {. Therefore if }
\end{aligned}
$$
$R_{1}<1$ then $a_{1} a_{2}-a_{3}>0, a_{1}>0$ and $a_{3}>0$. Hence, by using Routh-Hurwitz Criteria [14], all eigenvalues of $J\left(E^{*}\right)$ have negative real part whenever $R_{1}<1$. Thus, $E^{*}$ is locally asymptotically stable.

\subsection{Global stability}

First, we examine the global stability of $E_{0}$. Consider the Lyapunov function [15]:

$$
\begin{aligned}
L & =I+Z \\
\frac{d L}{d t} & =(-\mu+\alpha S) I-\mu Z .
\end{aligned}
$$

Since $E_{0} \in \Gamma$ then $S \leq \frac{B}{\mu}$ and we have $\frac{d L}{d t} \leq\left(-\mu+\frac{\alpha B}{\mu}\right) I-\mu Z \leq 0$ if $\frac{\alpha B}{\mu} \leq \mu$. It follows that $\frac{d L}{d t}<0$ if $\frac{\alpha B}{\mu^{2}} \leq 1 ;$ with $\frac{d L}{d t}=0$ if and only if $I=Z=0$. Hence, the only solution of system (1) in $\Gamma$ on which $\frac{d L}{d t}=0$ is $E_{0}$. Therefore, by LaSalle's Invariance Principle [15],every solution of system (1), with initial conditions in $\Gamma$, approaches $E_{0}$ as $t \rightarrow \infty$. Hence, $E_{0}$ is globally asymptotically stable and we can state the following theorem.

Theorem 4 If $\frac{\alpha B}{\mu^{2}} \leq 1$ then $E_{0}$ is globally asymptotically stable in $\Gamma$.

Next, we explore the global stability of $E^{*}$. Consider the Lyapunov function:

$$
\begin{aligned}
L & =\frac{1}{2}\left[\left(S-S^{*}\right)+\left(I-I^{*}\right)+\left(Z-Z^{*}\right)\right]^{2}, \\
\frac{d L}{d t} & =\left[\left(S-S^{*}\right)+\left(I-I^{*}\right)+\left(Z-Z^{*}\right)\right]\left[\frac{d S}{d t}+\frac{d I}{d t}+\frac{d Z}{d t}\right], \\
& =\left[\left(S-S^{*}\right)+\left(I-I^{*}\right)+\left(Z-Z^{*}\right)\right][B-\mu S-\mu I-\mu Z], \\
& =-\mu\left[\left(S-S^{*}\right)+\left(I-I^{*}\right)+\left(Z-Z^{*}\right)\right]^{2} \leq 0 .
\end{aligned}
$$

Hence, $E^{*}$ is globally stable.

Theorem 5 The positive (meme existence) equilibrium $E^{*}$ of system (1) is globally stable.

We summarize the result of this section as follows:

- If $R_{0}<1$, then $E_{0}$ exists only and it is both locally and globally asymptotically stable.

- If $R_{0}>1, R_{1}<1$, then $E_{0}$ is unstable, $E_{1}^{*}$ is locally asymptotically stable and globally stable.

- If $R_{0}>1, R_{1}>1$, then $E_{0}$ is unstable, $E_{1}^{*}$ and $E_{2}^{*}$ are globally stable if they exist. 


\section{Hopf bifurcation analysis}

In this section, we discuss Hopf bifurcation theory [14] for system (1) with $\alpha$ taken as the bifurcation parameter, and $R_{0}>1, R_{1}>1$.

From subsection 4.1 , the characteristic equation about $E^{*}$ is given by

$\lambda^{3}+a_{1} \lambda^{2}+a_{2} \lambda+a_{3}=0$.

By Routh-Hurwitz criteria a necessary and sufficient conditions for the eigenvalues to have negative real parts are:

$a_{1}>0, a_{3}>0$ and $a_{1} a_{2}>a_{3}$.

Thus, equation (3) will have two pure imaginary roots if and only if $a_{2}>0$ and $a_{1} a_{2}=a_{3}$ for some values of $\alpha$, say $\alpha^{*}$. Hence there is an interval containing $\alpha^{*}$, say $\left(\alpha^{*}-\varepsilon, \alpha^{*}+\varepsilon\right)$ for some $\varepsilon>0$ such that for $\alpha \in\left(\alpha^{*}-\varepsilon, \alpha^{*}+\varepsilon\right)$, equation (3) cannot have real positive roots.

For $\alpha=\alpha^{*}$, equation (3) can be factored into the form

$\left(\lambda^{2}+a_{2}\right)\left(\lambda+a_{1}\right)=0$,

which has three roots $\lambda_{1}=i \sqrt{a_{2}}, \lambda_{2}=-i \sqrt{a_{2}}$ and $\lambda_{3}=-a_{1}$. For $\alpha \in\left(\alpha^{*}-\varepsilon, \alpha^{*}+\varepsilon\right)$ the roots $\lambda_{1}, \lambda_{2}$ and $\lambda_{3}$ have in general the form:

$\lambda_{1}=\gamma(\alpha)+i \beta(\alpha)$.

$\lambda_{2}=\gamma(\alpha)-i \beta(\alpha)$.

$\lambda_{3}=-a_{1}(\alpha)$.

To apply Hopf bifurcation theorem [14] to system (1) we need to verify the condition

$\operatorname{Re}\left[\frac{d \lambda_{i}}{d \alpha}\right]_{\alpha=\alpha^{*}}=\gamma^{\prime}\left(\alpha^{*}\right) \neq 0, i=1,2$.

Substituting $\lambda_{1}=\gamma(\alpha)+i \beta(\alpha)$ and $\lambda_{2}=\gamma(\alpha)-i \beta(\alpha)$ into (3), and calculating the derivative, we obtain

$A(\alpha) \gamma^{\prime}(\alpha)-B(\alpha) \beta(\alpha)+C(\alpha)=0$

$B(\alpha) \gamma^{\prime}(\alpha)+A(\alpha) \beta(\alpha)+D(\alpha)=0$

Where

$A(\alpha)=3 \gamma^{2}(\alpha)+2 a_{1}(\alpha) \gamma(\alpha)+a_{2}(\alpha)-3 \beta^{2}(\alpha)$.

$B(\alpha)=6 \gamma(\alpha) \beta(\alpha)+2 a_{1}(\alpha) \beta(\alpha)$.

$C(\alpha)=a_{3}^{\prime}(\alpha)+a_{2}^{\prime}(\alpha) \gamma(\alpha)+a_{1}^{\prime}(\alpha) \gamma^{2}(\alpha)-a_{1}^{\prime}(\alpha) \beta^{2}(\alpha)$.

$D(\alpha)=a_{2}^{\prime}(\alpha) \beta(\alpha)+2 a_{1}^{\prime}(\alpha) \gamma(\alpha) \beta(\alpha)$.

Therefore $\operatorname{Re}\left[\frac{d \lambda_{i}}{d \alpha}\right]_{\alpha=\alpha^{*}}=-\left(\frac{A(\alpha) C(\alpha)+D(\alpha) B(\alpha)}{A^{2}(\alpha)+B^{2}(\alpha)}\right)_{\alpha=\alpha^{*}}, i=1,2$.

If we assume that $I^{*}<\frac{\mu}{\gamma-\beta}$ at $\alpha^{*}$, then $(\beta-\gamma) I^{*}+\mu>0$ and so $a_{1}\left(\alpha^{*}\right)>0$. If $A(\alpha) C(\alpha)+D(\alpha) B(\alpha) \neq 0$ then $\operatorname{Re}\left[\frac{d \lambda_{i}}{d \alpha}\right]_{\alpha=\alpha^{*}} \neq 0$ for $i=1,2$ and $\lambda_{3}=-a_{1}\left(\alpha^{*}\right) \neq 0$. Hence there is a Hopf bifurcation at $\alpha=\alpha^{*}$. We can now formulate the following:

Theorem 6 Suppose that $R_{0}>1, R_{1}>1$. If $I_{i}^{*}<\frac{\mu}{\gamma-\beta}$ where $i=1,2$, then system (1) may have a Hopf bifurcation as $\alpha$ passes through $\alpha^{*}$ emanating from equilibria $E_{1}^{*}$ and $E_{2}^{*}$ leading to periodic solutions for either $\alpha>\alpha^{*}$ or $\alpha<\alpha^{*}$ or at $\alpha=\alpha^{*}$.

\section{Numerical simulation}

To support the results obtained in this paper, numerical simulations of system (1) are illustrated using arbitrary values for the parameters with different initial conditions. Four different initial values are chosen such that $S+I+Z \leq$ $\frac{B}{\mu}:$

1. $\mathrm{S}(0)=0.3890, \mathrm{I}(0)=0.8540, \mathrm{Z}(0)=0.5360$,

2. $\mathrm{S}(0)=0.0010, \mathrm{I}(0)=0.5140, \mathrm{Z}(0)=0.3600$, 
3. $\mathrm{S}(0)=2.5485, \mathrm{I}(0)=0.0540, \mathrm{Z}(0)=0.8675$,

4. $\mathrm{S}(0)=0.8000, \mathrm{I}(0)=0.3154, \mathrm{Z}(0)=0.0250$.

First, we assign the parameters to the following values: $\beta=0.05, \mu=0.34, \gamma=0.015, \alpha=0.0125, \theta=$ $0.333, B=2$. Here $R_{0}=0.0720156<1$.

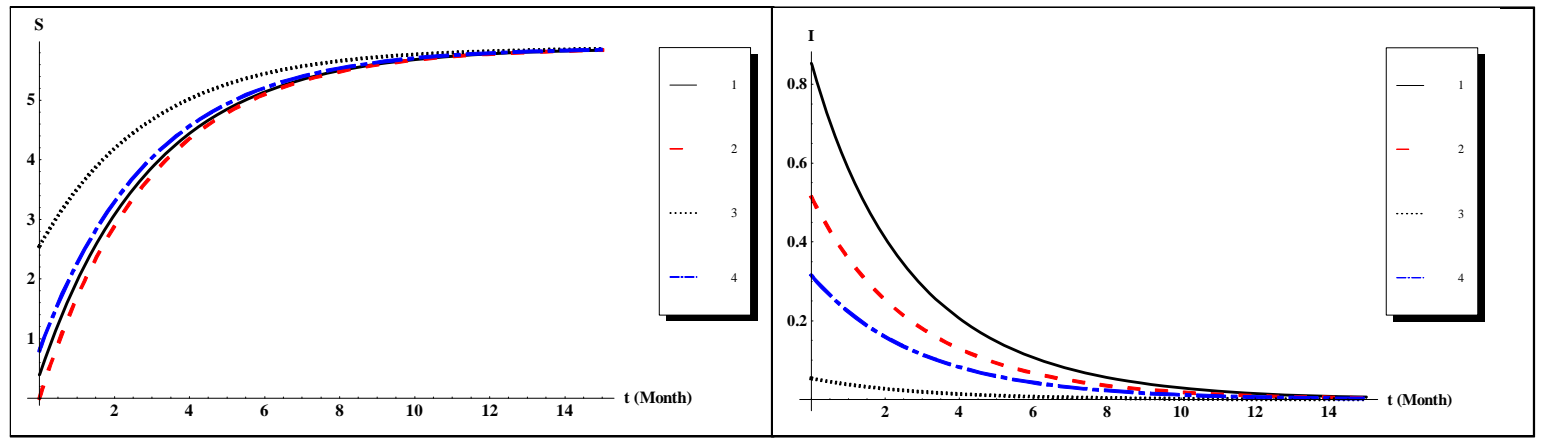

(a)

(b)

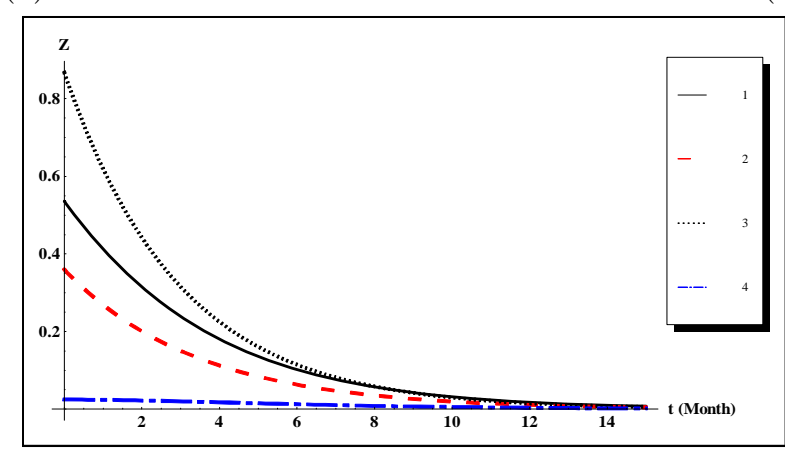

(c)

Fig. 1. Time plots of system 1 with different initial conditions for $R_{0}<1$. (a) Susceptible; (b) Spreaders; (c) Stiflers.

We see from Fig. 1(a) that the number of people who are susceptible to the meme increases as a function of time to approach the value of $S_{0}$ for the four sets of initial conditions. While Fig. 1(b,c) show that the number of spreaders and stiflers decreases as a function of time and approaches zero. Hence, for all sets of initial conditions the solution curves tend to the meme free equilibrium $E_{0}$ when $R_{0}<1$.

Second, for the same parameters except for: $\alpha=0.4, \theta=0.6$, we have $R_{0}=4.15225>1$.

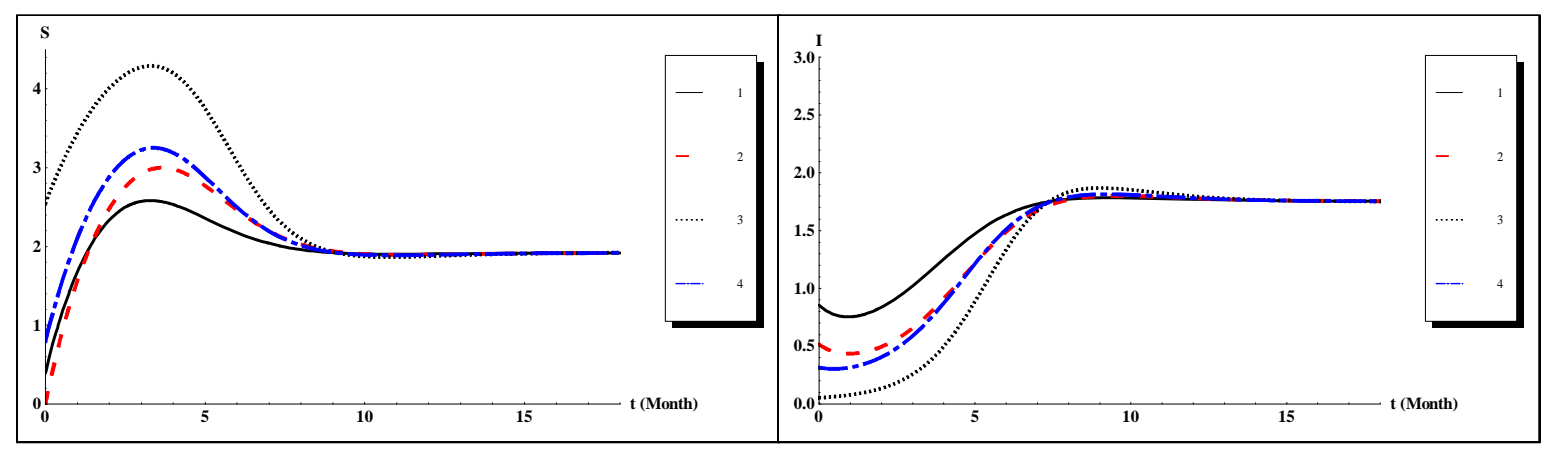

(a)

(b) 


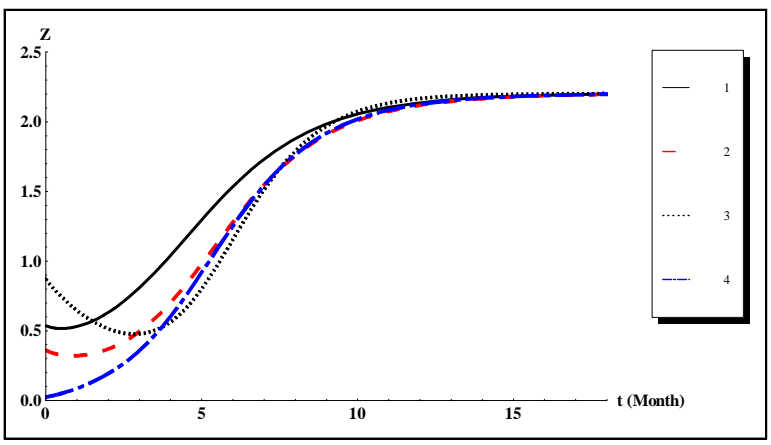

(c)

Fig. 2. Time plots of system 1 with different initial conditions for $R_{0}>1$. (a) Susceptible; (b) Spreaders; (c) Stiflers.

We see from Fig. 2(a) that for $R_{0}>1$ the number of susceptibles increases first then it starts to decrease and all solutions approach a certain value $S^{*}$. Also, Fig. 2(b) shows that the number of spreaders at the beginning of the meme increases rapidly, then it starts to decrease slightly and finally approaches a certain value $I^{*}$. A similar behavior for the stiflers is demonstrated in Fig. 2(c), they grow rapidly at first then they approach a certain value $Z^{*}$. Thus, for all sets of initial conditions the solution curves tend to the positive (meme existence) equilibrium $E^{*}$ when $R_{0}>1$.

\section{Discussion and conclusion}

In this paper, a nonlinear mathematical model of meme transmission is analyzed. Sufficient conditions have been given ensuring local and global stability of the meme free equilibrium point and the positive (meme existence) equilibrium point. We have shown that the model has two threshold values $R_{0}$ and $R_{1}$.

The meme free equilibrium $E_{0}$ is shown to be locally asymptotically stable whenever the basic reproduction number $R_{0}$ for the model is less than unity. If in addition the condition $\frac{\alpha B}{\mu^{2}} \leq 1$ is satisfied, then $E_{0}$ becomes globally asymptotically stable and the meme will disappear. The positive (meme existence) equilibrium $E^{*}$ exists when $R_{0}>1$ and it is shown to be locally asymptotically stable when $R_{1}$ is less than unity. We also proved that $E^{*}$ is globally stable which means that the meme persists. Finally, some numerical simulations is used to support these results.

This model can be extended in many ways to study different relations between the classes like for example, we can study the effect of stiflers becoming susceptibles again, which can happen when people tend to forget about the meme completely.

\section{References}

[1] K. Dietz, Epidemics and Rumors: A survey, Journal of the Royal Statistical Society, Series A (General), Vol. 130, No.4, (1967) 505-528.

[2] V. Cane, A Note on the size of epidemics and the number of people hearing a rumor, Journal of the Royal Statistical Society, Series B (Methodological), Vol. 28, No. 3, (1966) 487-490.

[3] K. Thompson, R. Estrada, D. Daugherty, and A. Cintron-Arias, A deterministic approach to the spread of rumors, Working paper, Washington, DC, USA, (2003).

[4] L. Bettencourt, A. Cintron-Arias, D. Kaiser, and C. Castillo-Chavez, The power of a good idea: Quantitative modeling of the spread of ideas from epidemiological models, Physica A, 364 (2006) 513-536.

[5] K. Kawachi, Deterministic models for rumors transmission, Nonlinear analysis: Real world applications, 9 (2008) 19892028.

[6] K. Kawachi, M. Seki, H. Yoshida, Y. Otake, k. Warashina and H. Ueda, A rumor transmission model with various contact interactions, Journal of theoretical biology, 253 (2008) 55-60. 
[7] J. Piqueira, Rumor propagation model: an equilibrium study, Hindawi Publishing Corporation Mathematical Problems in Engineering, Vol. (2010), Article ID 631357, doi:10.1155/2010/631357.

[8] W. Huang, On rumor spreading with skepticism and denial, Working paper, (2011).

[9] L. Wang, B. Wood, An epidemiological approach to model the viral propagation of memes, Applied Mathematical Modelling 35 (2011) 5442-5447.

[10] L. an Huo, P.Huang, and C.X. Guo, Analyzing the dynamics of a rumor transmission model with incubation, Hindawi Publishing Corporation Discrete Dynamics in Nature and Society, Vol. (2012), Article ID:328151, doi:10.1155/2012/328151.

[11] L. Zaho, Q. Wang, J. Cheng, D. Zhang, T. Ma, Y. Chen, and J. Wang, The impact of authorities media and rumor dissemination on the evolution of emergency, Physica A: Statistical Mechanics and its Applications, Volume 391, Issue 15 (2012) 3978-3987.

[12] F.Brauer, P.van den Driessche, and J.Wu, Mathematical Epidemiology, Springer-Verlag, (2008).

[13] L.Perko, Differential Equations and Dynamic Systems, Springer Verlag,(1991).

[14] L.Edelstein- Keshet, Mathematical Models in Biology, SIAM, (2005).

[15] M.W.Hirsch, S.Smale, and R.L.Devaney, Differential equations, dynamical systems and an introduction to chaos, Elsevier Academic press,(1974). 